\title{
ON FINITELY PRESENTED METABELIAN GROUPS
}

\author{
BY GILBERT BAUMSLAG ${ }^{1}$ \\ Communicated by George Seligman, September 8, 1971
}

1. The object of this note is to announce the following:

THEOREM. Every finitely generated metabelian group can be embedded in a finitely presented metabelian group.

This theorem should be compared with the example of a 3-generator, 3-relator group with a derived group which is free abelian of infinite rank [2]. It serves to illustrate, in particular, that the normal subgroup structure of (even) finitely presented (metabelian) groups is much more complex than one might have thought.

2. Sketch of the proof. Let $G$ be a finitely generated metabelian group.

The first step in the proof is to embed $G$ in a factor group $W / N$ of the wreath product $W$ of two finitely generated abelian groups. This embedding is chosen in such a way that $N$ is contained in the base group of $W$.

The second, and in fact the main step in the proof, is to embed $W$ in a finitely presented metabelian group $\hat{W}$ in such a way that the normal closure $\hat{N}$ of $N$ in $\hat{W}$ meets $W$ in $N$ :

$$
\hat{N} \cap W=N .
$$

It follows that $G$ is embedded in $\hat{W} / \hat{N}$. It follows immediately from a theorem of Philip Hall [1] that every factor group of a finitely presented metabelian group is finitely presented. Thus $G$ has been embedded in the finitely presented metabelian group $\hat{G} / \hat{N}$.

3. There are continuously many 2-generator center-extended-bymetabelian groups (P. Hall [1]). This means that there are 3-step solvable (indeed, center-extended-by-metabelian) groups, on 2 generators, that cannot be embedded in any finitely presented group whatsoever, let alone in a finitely presented solvable group of derived length 3 .

\section{REFERENCES}

1. P. Hall, Finiteness conditions for soluble groups, Proc. London Math. Soc. (3) 4 (1954), 419-436. MR 17, 344.

2. G. Baumslag, $\boldsymbol{A}$ finitely presented metabelian group with a free abelian derived group of infinite rank, Proc. Amer. Math. Soc. (submitted).

DePartment of Mathematics, Rice University, Houston, TeXas 77001

AMS 1970 subject classifications. Primary 20F05.

Key words and phrases. Finitely presented metabelian group.

${ }^{1}$ Support from the National Science Foundation is gratefully acknowledged. 